SUBJECT AREAS:

SUB-WAVELENGTH

OPTICS

NANOPHOTONICS AND

PLASMONICS

NANOPARTICLES

SCIENTIFIC REPORTS:

$3: 3047$

DOI: $10.1038 /$ srep03047

Published:

25 October 2013

Updated:

27 February 2014

\section{ERRATUM: Selective Optical Assembly of Highly Uniform Nanoparticles by Doughnut-Shaped Beams}

Syoji Ito, Hiroaki Yamauchi, Mamoru Tamura, Shimpei Hidaka, Hironori Hattori, Taichi Hamada, Keisuke Nishida, Shiho Tokonami, Tamitake Itoh, Hiroshi Miyasaka \& Takuya lida

The incorrect versions of Supplementary Movies 2 and 3 were published with the original Article. In the original versions of Supplementary Movies 2 and 3 the size of the background colour maps were modified during the file conversion process. The numerical results contained in the videos were correct. 